\title{
Research on the Evaluation Mode of 95\% Probability Value in the National Standard GB/T 14549-93
}

\author{
Jiang Zeng, Wei-Wei Sun* and De-Hua Huang \\ College of Electric Power, South China University of Technology, Guangzhou 510640, Guangdong, China
}

\begin{abstract}
The national standard GB/T 14549-93 stipulated 95\% probability value as the judgment basis of the harmonic pollution degree, many problems turned up over the past ten years. Based on a study of several typical cases, this paper points out that the evaluation mode may carry out unfair evaluation results, destroy the consistency, and continuity of the measurement data, and analyzes the cause of the evaluation mode defects. Then, a new evaluation index and a data recording method were proposed and validated by experimental and simulation results to solve the existing problems quite well. The experimental and simulation results show that the new evaluation index provides a workable idea and suggestion for the national standard revision, and it should be applied to judge whether harmonic is excessive. The proposition of the new evaluation mode and index will benefit to perfecting harmonic management system and maintaining social equity.
\end{abstract}

Keywords: Power system; harmonic; national standard; probability value; evaluation index

\section{Introduction}

In recent years, harmonic has constituted more and more threat to the security, stability and economic operation of power grid and influenced the electrical environment. The national standard GB/T 14549-93 [1] was issued to control the harmonic pollution, and it stipulated the harmonic voltage limit, the harmonic current distribution index of users, measurement instruments, measurement methods and related calculation for different voltage level $(380 \mathrm{~V} \sim 220 \mathrm{kV})$ in public supply network. The rationality of the harmonic standard has vital relationship to the technology and economic benefits of both the power grid and utilization and was always concerned, also many problems appeared in the 10 years.

According to the related basic concept of harmonic in the electromagnetic compatibility and power quality, Reference [2] clarified some current national standard problems. Also, Reference [3] pointed out several major problems in the national standard, such as harmonic voltage limit, harmonic current distribution index of users, harmonic impedance processing and harmonic measurement window width, and it exemplified the urgency and necessity of revising the current national standard. Reference [4] pointed out that the current national standard stipulated only the measurement method of some simple connection and operation modes, but not complex conditions and operation modes. Reference [5] pointed out that the provisions about changed load measurement did not involve the choice of measurement sampling window, and therefore it leads to some original defects and needs further specification. In recent years, research on the harmonic national standard defects made great progress. Reference [6] and Reference [7] studied the harmonic influence to the electricity measurement and both the power grid and utilization; Hilbert transform, improved Fourier series, particle swarm and neural network algorithm were applied to harmonic analysis and measurement in Reference [8], Reference [9] and Reference [10], they pointed out some harmonic national standard defects and put forward corresponding solutions or thinking.

\footnotetext{
* Manuscript received June 10, 2012; revised July 24, 2012.

Corresponding author. Tel.: +86-15013054292; E-mail address: sww689021@163.com.
} 
This paper discusses the evaluation mode of $95 \%$ probability value as whether harmonic is excessive and it points out that the evaluation method may lead to the evaluation injustice about harmonic pollution, and it destroys the data consistency and continuity, combined with the harmonic measurement provisions in the national standard. Finally, this paper presents a new method, based on analyzing why the defects exist. The method is used for harmonic measurement and to form a new evaluation index. Also, this paper proves that the new method can effectively solve the evaluation method defects of $95 \%$ probability value.

\section{Current National Standard}

The harmonic voltage limit of different voltage levels is the basis of the national standard. The regulation [11] of harmonic voltage limit in the GB/T14549-93 is shown in Table 1. Considering the harmonic volatility, the current national standard rules that the largest $95 \%$ probability value of different phases in measurement time should be taken as the harmonic measurement data and the judgment whether harmonic is excessive. For practicality convenience, it is suggested that $95 \%$ probability value may be approximately selected through wiping out the $5 \%$ front great value of the harmonic data arranged in descending order and taking the maximum residual values.

Table 1. Harmonic voltage (phase voltage) limit in public supply network in China

\begin{tabular}{cccc}
\hline \multirow{2}{*}{ Nominal voltage $(\mathrm{kV})$} & \multirow{2}{*}{ THDu (\%) } & \multicolumn{2}{c}{ HRUh (\%) } \\
\cline { 3 - 4 } & & Odd & Even \\
\hline 0.38 & 5 & 4 & 0.38 \\
\hline $6 \sim 10$ & 4 & 3.2 & $6 \sim 10$ \\
\hline $35 \sim 66$ & 3 & 2.4 & $35 \sim 66$ \\
\hline 110 & 2 & 1.6 & 110 \\
\hline
\end{tabular}

According to the advice of CIGRE 36-05 [12], to distinguish transient phenomena and harmonic, we should take the average valve in $3 \mathrm{~s}$ as the measurement result. Due to the widely used digital harmonic analyzers, the national standard gives the recommended type according to discrete sampling like that:

$$
U_{h}=\sqrt{\frac{1}{m} \sum_{k=1}^{m}\left(U_{h k}\right)^{2}}
$$

where $m$ is the measurement times in $3 \mathrm{~s}, m \geq 6, U_{h k}$ is the party root value of the $\mathrm{h}$ harmonic measured in the first $k$ time in $3 \mathrm{~s}$.

The national standard GB/T19862-2005 rules that a basic record cycle of harmonic measurement is $3 \mathrm{~s}$, and a storage record cycle is $3 \mathrm{~min}$. The basic record is formed by root mean square method, and the storage record is formed by the biggest valve principle, namely taking the maximum value of 60 basic records within 3 min as a storage record.

In addition, due to the discrete Fourier transform (DFT), taking a power frequency harmonic sampling can only analyze integer-times harmonic. But between harmonics widely exist in power grid, to analyze between harmonics and avoid its influence to single frequency sampling, IEC61000-4-7:2002 stipulates the measurement sampling window $0.2 \mathrm{~s}$. The national standard did not make a clear regulation, but most measurement instruments were designed in this way.

\section{Defects of 95\% Probability Value}

The evaluation mode of $95 \%$ probability value has not made limits for the residual $5 \%$ data. In fact, the unusual or accident tends to happen in overweight period. IEC61000-3-6 and the United States, Russia standard [13]-[15] stipulated the normal and biggest allowable value. The evaluation mode of 95\% probability value concerns only whether harmonic is excessive, but not the pollution degree of the same 95\% probability value but different harmonic distribution. Furthermore, it destroys the consistency and continuity of the measurement data. This paper will take the harmonic data of two $6 \mathrm{kV}$ bus in a large oil producer to explain the evaluation mode defects of $95 \%$ probability value from several different aspects. 


\subsection{Operation condition change}

The $24 \mathrm{~h}$ time evolution diagram of harmonic voltage data in bus 1 is shown in Fig. 1. According to the current evaluation mode, all data in Fig. 1 will be arranged in descending order. As shown in Fig. 2, the intersection of the vertical line and the curve is the $95 \%$ probability value of 5 th harmonic voltage distortion rate in A phase, it is $2.16 \%$. When the operation condition changed slightly, its harmonic data may change correspondingly, as shown in Fig. 3, and its 95\% probability value was $4.72 \%$.

The two curves in Fig. 2 and Fig. 3 are almost the same, only 20 more different digits exist, it may be caused by the equipment running time difference in actual working condition. The harmonic pollution degree should be very small, but their evaluation results are very different, obviously, it is unfair.

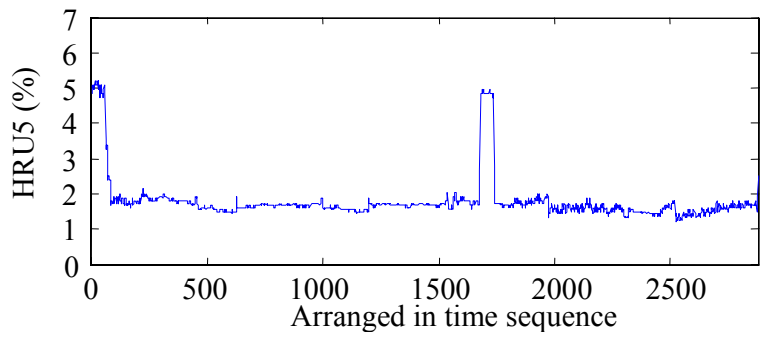

Fig. 1 . The $24 \mathrm{~h}$ time evolution diagram of harmonic voltage data in bus 1

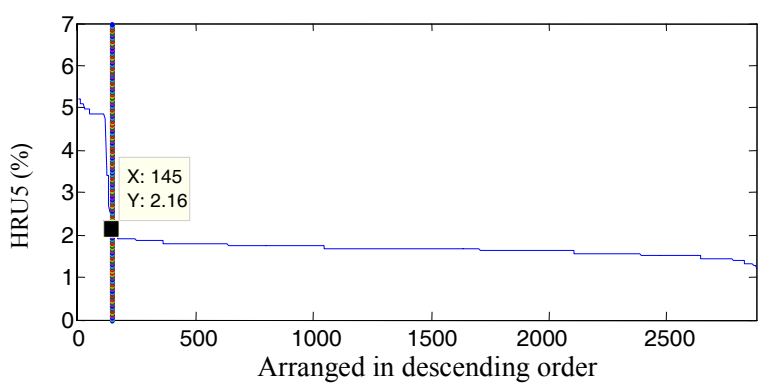

Fig. 2. The descending arrangement figure of harmonic voltage data in bus 1

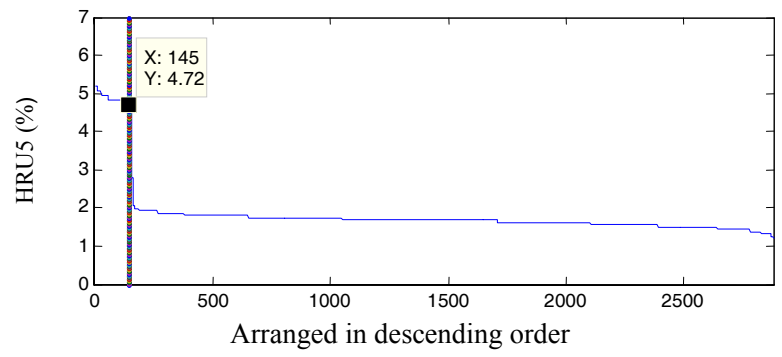

Fig. 3. The descending arrangement figure of harmonic voltage data in bus 1 (small changes in operating condition)

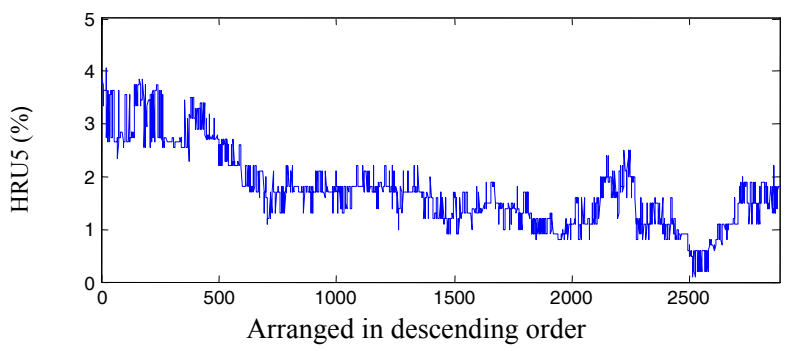

Fig. 4. The $24 \mathrm{~h}$ time evolution diagram of harmonic voltage data in bus 2

\subsection{Different harmonic fluctuation}

The $24 \mathrm{~h}$ time evolution diagram of harmonic voltage data in bus 2 is shown in Fig. 4 . All data will be arranged in descending order, and the curve in figure 2 will be drawn along with Fig. 5, its 95\% 
probability value is $3.56 \%$. As shown in Fig. 5, the harmonic voltage distortion rate of bus 2 is not excessive, but its $95 \%$ probability value is bigger than that of bus 1, caused by small fluctuation in bus 2 . Obviously, harmonic pollution in bus 1 is more serious, the evaluation result is unreasonable. It can be seen that taking $95 \%$ probability value directly in the top $5 \%$ and ignoring its impact is not appropriate.

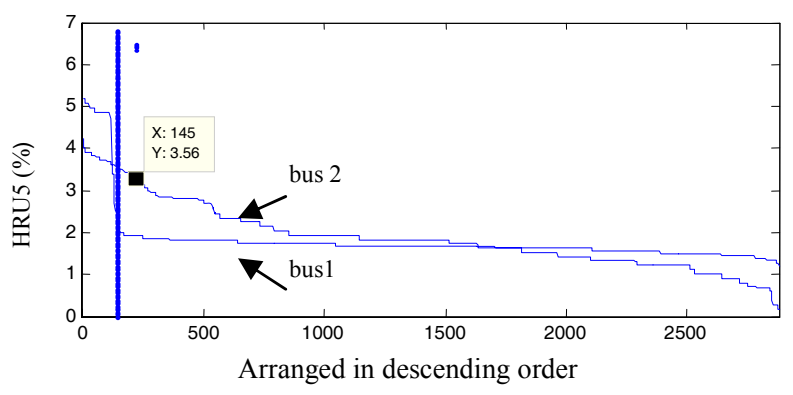

Fig. 5. The descending arrangement figure of harmonic data in bus 2

\subsection{Different measurement method}

When we adopt a long-term and continuous measurement, using a short storage record will produce a large amount of data, it is very inconvenient in actual operation, so using a long storage record on the basis of basic record is widely adopted. RMS method is recommended to keep records in IEC61000-4-30, and the record cycle is $3 \mathrm{~s}, 10 \mathrm{~min}$ or $2 \mathrm{~h}$. The national standard stipulates the record cycle $3 \mathrm{~min}$.

Using different record cycle and formation method is actually the different storage and processing of all the observations. If the sampling measurement window is $0.2 \mathrm{~s}$, the $24 \mathrm{~h}$ operation conditions can be simulated by 432000 random data meeting normal distribution, as shown in Fig. 3. The $95 \%$ probability value of all the observations is $6.647 \%$. According to $3 \mathrm{~s}, 10 \mathrm{~min}$ and $2 \mathrm{~h}$ record cycle recommended by IEC61000-4-30, the $95 \%$ probability value is respectively $5.511 \%, 5.130 \%$ and $5.106 \%$ According to 3 min record cycle by the national standard, the $95 \%$ probability value is $5.904 \%$. It can be seen that different record cycle and formation method of storage records will produce deviated evaluation results, the data consistency will be destroyed.

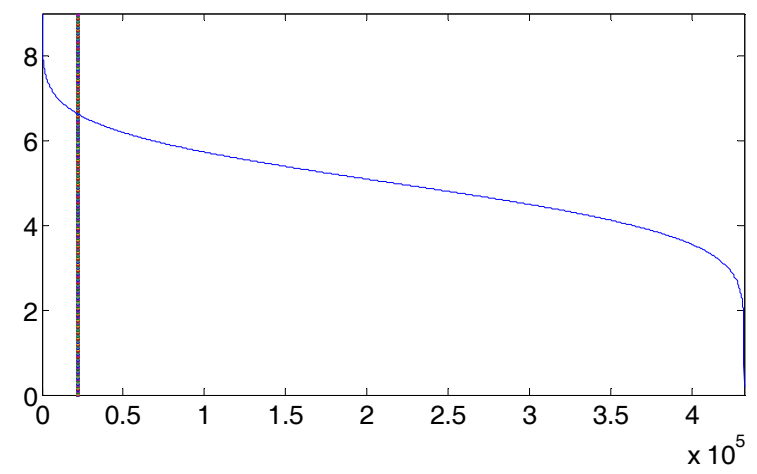

Fig. 6. Packet calculation and direct calculation comparison of harmonic data

The root cause of such deficiency is that the data processing algorithm in the two stages is different. During the harmonic measurement, the record value is formed by party root method or the biggest principle, while, during the computation, the $95 \%$ probability value is directly the result of arranging the measurement data in descending order. In actual production, monitoring a running equipment using different measurement instruments with the same precision, different record cycles will result in different evaluation results, obviously, it is not reasonable. Considering the influence of the measurement window and record cycle difference, the GB/T19862-2005 made mandatory provisions, but it can't eliminate the evaluation mode defects of $95 \%$ probability value and influences the measurement instrument flexibility. 


\section{New Evaluation Method}

\subsection{New evaluation mode and its parameters}

In view of the evaluation mode deficiency of $95 \%$ probability value, new evaluation index should be considered. The new record form and evaluation mode should meet the following requirements:

1) The evaluation index should be continuous. Namely, the evaluation index won't change dramatically due to small measurement fluctuation.

2) The evaluation index should be consistent. Namely, the evaluation result won't change dramatically due to different measurement window, time, record cycle.

3) The evaluation index can reflect harmonic pollution degree more accurately. The evaluation index should reflect the harmonic pollution degree and meet the actual measurement need. From the practice of long-term harmonic management, faults diagnosis, and pollution control, harmonic damage often happen in overweight period, the evaluation index must reflect the damage in overweight period. And the longterm harmonic pollution loss can be limited or evaluated by RMS index.

Considering the requirements, this paper proposes the high time RMS method to keep records, such as type 2. During the harmonic data processing, type 3 will be used to calculate the new evaluation index (recorded as A), A is used to judge whether harmonic is excessive instead of the $95 \%$ probability value.

$$
U_{h i}=\sqrt[n]{\frac{1}{m} \sum_{k=1}^{m}\left(U_{h k}\right)^{n}}
$$

where $U_{h i}$-The first $i$ record value of the $h$ harmonic; $m$ - measurement times during the basic record, $m \geq 6, U_{h k}$-The party root value of the $h$ harmonic measured in the first $k$ time during the basic record;

$$
A=\sqrt[n]{\frac{1}{N} \sum_{i=1}^{N}\left(U_{h i}\right)^{n}}
$$

where $A$ is the new evaluation index and $N$ is the record data number.

The new evaluation index will not generate large deviation due to tiny changes in the overall data and it ensures the measurement data continuity. Also, the same formula form is used in the preservation and processing stage, and the evaluation index A consists of all information in the measurement and won't generate deviated evaluation results due to different record cycle. The new evaluation index is consistent.

When $n$ is a large number (such as 8,16 and etc), the calculation result will be led by the larger data. The new evaluation index fully highlights the harmonic pollution influence and it can solve the current evaluation mode defects. Because the new algorithm is related to the high time calculation of large quantities of data, the calculation speed is concerned. To adapt to the microcomputer processing and save calculation time, $n$ is set power of 2 , and $a^{4}$ can be got by two times multiplication of $a^{2}$, also $a^{6}$ can be got by the multiplication of $a^{4}$ and $a^{2}$. In this way, the calculation time can be effectively saved. Through the random data test of different distribution and the same distribution with different variance, we take $n$ as 16 relatively appropriate. The expression of the new evaluation method can be further identified as:

$$
A=\sqrt[16]{\frac{1}{N} \sum_{i=1}^{N}\left(U_{h i}\right)^{16}}
$$

\subsection{Effectiveness validation of the new evaluation mode}

1) The harmonic pollution of bus 1 was evaluated using the new evaluation mode, and the evaluation result is $4.05 \%$. When the operation condition changed a little, the evaluation result is $4.10 \%$, shown in Table 2. The above two evaluation results are appropriate.

2) When the harmonic pollution in bus 2 was evaluated using the new evaluation index, the evaluation result was $3.18 \%$, it was not excessive and less than the evaluation result in bus 1 , and it was reasonable. 
3) When the data in Fig. 6 was calculated directly or separately, the evaluation results are both $6.13 \%$. The new evaluation mode contains all the measurement information and it is of data consistency and solves the evaluation mode defects of $95 \%$ probability value in processing packet data.

In order to avoid the huge amount of data in a long-term and continuous measurement, we can adopt different record cycles according to different operation conditions and requirements, without worrying about the rationality of the evaluation results.

Table 2. Different Harmonic voltage (phase voltage) limit in bus 1 and bus 2 by different evaluation modes

\begin{tabular}{cccc}
\hline Evaluation mode & Bus 1 & Little change in operation condition in bus 1 & Bus 2 \\
\hline 95\% probability value & 2.16 & 4.72 & 3.56 \\
\hline High time RMS & 4.05 & 4.10 & 3.18 \\
\hline
\end{tabular}

\section{Conclusions}

The national standard GB/T14549-93 has been applied for more than 16years and different problems and defects appeared during its implementation. So its revision is urgent to meet the harmonic management requirement in new times. In order to solve the evaluation mode defects of $95 \%$ probability value, this paper puts forward the new evaluation mode of high time RMS. Through verification, the new evaluation mode is feasible and it makes harmonic pollution evaluation more accurate and reasonable, and it can solve the evaluation mode deficiency of $95 \%$ probability value.

It must be pointed out that the new evaluation mode is still in the theory research stage and needs more inspection of the theoretical arguments and measurement data. But it provides a train of thought and reference for the national standard revision, and it benefits to maintaining the social justice.

\section{References}

[1] Jiang JX. The national standard GB/T 14549-93-Quality of electric energy supply Harmonics in public supply network profile. Power Capacitors, 1999; (02):22-25.

[2] Qu T, Wu JC, Lv RY. Clarification of some questions concerning harmonics in public supply network and electromagnetic compatibility. Electric Power, 2001; 34(3):29-32.

[3] Lin HX. Some problems in national standard for harmonics in public supply network. Power System Technology, 2003; 27(1):65 70.

[4] $\mathrm{Wu} \mathrm{W}$, Chen Y. Engineering application of national standards of harmonics. East China Electric Power, 2007; 35(12):113115.

[5] The Voltage Current And Frequency Standardization Technical Committee. Power Quality National Standard Application Guide. Beijing: Standard Press of China, 2009.

[6] Zhang YJ, Shi H. Harmonic error modifying of electric energy meter considering harmonic responsivity, Power System Protection and Control, 2009; 37(22):58-61.

[7] Huang YC. Analysis and discussion of power harmonics influencing the electric energy computation, Power System Protection and Control, 2009; 37(10):123-124.

[8] Zhou XC, Liu J, Zheng W, Zhi Y. Harmonics analysis based on wavelet -mathematical morphology and Hilbert, Power System Protection and Control, 2009; 37(16):20-23.

[9] Tan SS, Liu QJ. Hybrid algorithm for interharmonics measurement based on particle swarm optimization and neural network, Power System Protection and Control, 2011; 39(2):18- 22.

[10] Xu ZN, Lv FC. A high accuracy harmonic analysis algorithm based on modified Fourier series, Power System Protection and Control, 2011; 39(10):27-30.

[11] Quality of Electric Energy Supply Harmonics in Public Supply Network. GB/T 14549-93

[12] Working Group 36.05. Harmonics, characteristic parameters, methods of study, estimates of existing values in the network. Electra, 1981(77):35-54.

[13] EMC Part 3: Limits-Section6: Assessment of Emission Limits for Distorting Loads in MV and HV Power Systems. IEC 610003-6, 1996.

[14] IEEE Recommended Practices and Requirements for Harmonic Control in Electrical Power System. IEEE Std 519-1992.

[15] Electric Energy, Electromagnetic Compatibility of Technical Equipment Power Quality Limits in Public Electrical Systems. GOST 13109-1997. 